\title{
Synergistic Effect of Fullerene-Capped Gold Nanoparticles on Graphene Electrochemical Supercapacitors
}

\author{
Virginia Yong ${ }^{*}$, H. Thomas Hahn \\ Department of Materials Science and Engineering, University of California, Los Angeles, USA \\ Email: "hyong@ucla.edu
}

Received January 1, 2013; revised January 28, 2013; accepted February 4, 2013

\begin{abstract}
We report the synthesis of graphene/fullerene-capped gold nanoparticle nanocomposite film which was used to construct supercapacitor electrodes. The fullerene-based self-assembled monolayers on gold nanoparticles (AuNPs) were attained via the fullerene $\left(\mathrm{C}_{60}\right)$-gold interaction. The fullerene-capped AuNPs effectively separated the graphene sheets preventing aggregation. A synergistic effect was observed - the specific capacitance of graphene/fullerene-capped AuNP electrode is $197 \mathrm{~F} / \mathrm{g}$, which is higher than that of graphene electrode $(31 \mathrm{~F} / \mathrm{g})$, graphene/AuNP electrode $(126$ $\mathrm{F} / \mathrm{g})$, and graphene/fullerene electrode $(118 \mathrm{~F} / \mathrm{g})$. The results render a novel route of synthesis and modification of graphene-based materials for the construction of electrochemical energy storage devices.
\end{abstract}

Keywords: Fullerenes; Gold Nanoparticles; Graphene; Supercapacitors; Energy Storage; Electrochemistry; Self-Assembly; Nanocomposites

\section{Introduction}

Supercapacitors, also called electrochemical capacitors or ultracapacitors, are considered as a promising candidate for energy storage because of their high power capability, fast charge/discharge rates, long cycle life, and low maintenance cost. The discovery of closed zero-dimensional (0D) buckminsterfullerenes $\left(\mathrm{C}_{60}\right)$ in 1985 [1] and most recently the experimental isolation of $2 \mathrm{D}$ graphene in 2004 [2] by micromechanical cleavage of highly oriented pyrolytic graphite (HOPG) have stimulated much experimental and theoretical research, owing to their unusual structure and extraordinary electronic, transport and optical properties [3]. There has been a tremendous amount of research aimed at developing new carbon-based materials with novel useful applications [4]. Graphene is considered to be an excellent electrode material for supercapacitors due to its high electrical conductivity, high surface area, great flexibility, excellent mechanical properties, and rich chemistry [5]. The surface area of a single graphene sheet is $2630 \mathrm{~m}^{2} / \mathrm{g}$, substantially higher than values derived from BrunauerEmmett-Teller (BET) surface area measurements of activated carbons used in current electrochemical double layer capacitors [6]. However, aggregation of isolated graphene sheets during drying graphene dispersions leads to a loss of its ultrahigh surface area advantage as a two-dimensional nanomaterial, resulting in low capacitance.

"Corresponding author.
Self-assembled monolayers (SAMs) of alkane thiols on gold nanoparticles (AuNPs) have been the most extensively studied systems due to the well-defined organosulfur adsorption chemistry on $\mathrm{Au}$ surfaces [7]. Thiols, thiol esters, or disulfides are well-known to be absorbed onto a gold surface to form a SAM, owing to the strong S-Au bond strength $(\sim 180 \mathrm{~kJ} / \mathrm{mol})$ [8]. SAMs of fullerenes have attracted increasing interest in many emerging assembly strategies. Previous theoretical and experimental studies have shown that $\mathrm{C}_{60}$ can be bound tightly to the gold surface by van der Waals interactions, with a $\mathrm{C}_{60}$-gold binding energy of $\sim 1 \mathrm{eV}$ [9]. The $\mathrm{C}_{60}-\mathrm{Au}$ bond strength has been reported to be $\sim 200 \mathrm{~kJ} / \mathrm{mol}$, [10] which is comparable with the strength of the S-Au bond. They are thus compatible to the thiol-based SAMs on AuNPs which have been extensively studied and exploited in many applications. Recently, AuNPs of $10 \mathrm{~nm}$ were used as an efficient spacing material for electrochemically reduced graphene oxide, which greatly increased the capacitance from $4.99 \mathrm{~F} / \mathrm{g}$ (when no spacers are used) to $174.2 \mathrm{~F} / \mathrm{g}$ (when gold nanospacers are introduced) [11]. Here, we report a novel route of synthesis and self-assembly processes of fullerene-capped AuNPs which are used as the spacing material of graphene sheets for supercapacitor electrodes.

\section{Experiment}

Hydrogen tetrachloroaurate trihydrate $\left(\mathrm{HAuCl}_{4} \cdot 3 \mathrm{H}_{2} \mathrm{O}\right)$, dodecylamine (DDA), tetrabutylammonium borohydride 
(TBAB), and toluene were purchased from Sigma-Aldrich. Didodecyldimethylammonium bromide (DDAB) was purchased from TCI America. Hydrazine monohydrate $\left(\mathrm{N}_{2} \mathrm{H}_{4} \cdot \mathrm{H}_{2} \mathrm{O}\right)$ was purchased from Fluka. Fullerene derivative [6,6]-phenyl-C61 butyric acid methyl ester (PCBM) was purchased from Nano-C. All reagents and solvents were used without further purification.

AuNPs were synthesized by a seeded growth method [12]. Specifically, DDAB stock solution was first prepared by dissolving $925 \mathrm{mg}$ DDAB in $20 \mathrm{ml}$ toluene. $\mathrm{HAuCl}_{4} \cdot 3 \mathrm{H}_{2} \mathrm{O}(50 \mathrm{mg})$ and DDA $(450 \mathrm{mg})$ were added to $12.5 \mathrm{ml}$ of the stock solution and sonicated until dissolved. Gold (III) was then reduced by dropwise addition of $125 \mathrm{mg}$ TBAB in $5 \mathrm{ml}$ of the DDAB stock solution under vigorous stirring. Thus prepared seed (4 nm NPs) solution was aged for 24 hours. The growth solution was prepared by adding $14 \mathrm{ml}$ of the aged seed solution to $100 \mathrm{ml}$ of toluene containing $400 \mathrm{mg} \mathrm{HAuCl} \mathrm{H}_{4} \cdot 3 \mathrm{H}_{2} \mathrm{O}, 2.00$ g DDAB, and 3.7 g DDA. Finally, $262 \mu \mathrm{l}$ hydrazine dissolved in $40 \mathrm{ml}$ of the stock solution was added dropwise to the growth solution under vigorous stirring. Thus prepared AuNPs have diameters of $5.6 \pm 0.6 \mathrm{~nm}$.

A $50 \mathrm{ml}$ sample of the freshly prepared DDA-capped AuNP solution (ca. $7.25 \mathrm{mM}$ ) was quenched with $250 \mathrm{ml}$ of methanol to give NP precipitate. The resulting NP precipitate was collected and sonicated in a $3.5 \mathrm{mM}$ toluene solution of PCBM with desired proportions [typically, AuNPs to PCBM in 2:1 (wt/wt)] for $1 \mathrm{hr}$. The calculated proportions for the formation of a single PCBM monolayer-protected AuNPs in solution and in solid film are 8:1 and 10:3 (AuNPs to PCBM in wt/wt), respectively. A larger amount of PCBM was used owing to the solubility of PCBM in toluene. The as-prepared PCBM-capped AuNPs were then purified by precipitation with a large amount of methanol $(90 \% \mathrm{v} / \mathrm{v})$ followed by several cycles of sonication in toluene, washed with methanol, and re-dispersed in toluene.

Graphene sheets were prepared using the chemical vapor deposition method [13]. The graphene-based nanocomposites (PCBM-capped AuNPs/graphene, AuNPs/graphene, and PCBM/graphene) were synthesized with graphene at a constant fraction of $25 \mathrm{wt} \%$. Typically, 0.15 $\mu \mathrm{g}$ of graphene was mixed with $0.45 \mu \mathrm{g}$ of PCBMcapped AuNPs (in control experiments, AuNPs or PCBM was used instead of PCBM-capped AuNPs). A $1 \mu \mathrm{L}$ mixture was deposited on the glassy carbon working electrode surface and dried.

Dynamic light scattering (DLS) was performed on a Beckman Coulter particle size analyzer. X-ray photoelectron spectroscopy (XPS) analyses were conducted using an Omicron ESCA probe operated in vacuum at around $5 \times 10^{-10}$ torr $(1$ torr $=133.322 \mathrm{~Pa})$, which was equipped with an EA125 energy analyzer. Photoemission was stimulated by a monochromated $\mathrm{Al} \mathrm{K} \alpha$ radiation $(1486.6 \mathrm{eV})$ with an operating power of $300 \mathrm{~W}$. The takeoff angle was set at $45^{\circ}$. A low-energy electron flood gun was employed for charge neutralization. High resolution TEM (HRTEM) imaging, electron energy loss spectrometry (EELS), and scanning TEM (STEM) studies were carried out using a JEOL $2100 \mathrm{~F}$ field emission gun electron microscope operated at $200 \mathrm{kV}$ accelerating voltage. The instrument was equipped with a Gatan slowscan charge-coupled device (CCD) camera, and Gatan EELS attachments. Cyclic voltammetry (CV) was performed on a Bioanalytical Systems E2-001000 electrochemical workstation. A three-electrode cell was employed for electrochemical measurements, where an $\mathrm{Ag} /$ $\mathrm{AgCl}$ electrode and a $\mathrm{Pt}$ wire were used as the reference and counter electrode, respectively. The cyclic voltammograms were obtained in $0.5 \mathrm{M} \mathrm{KCl}$ buffer at room temperature at scan rates of 25,50 , and $100 \mathrm{mV} / \mathrm{s}$.

\section{Results and Discussion}

AuNPs stabilized by SAMs of fullerene derivative PCBM (Figure 1(a)) were prepared as described in the

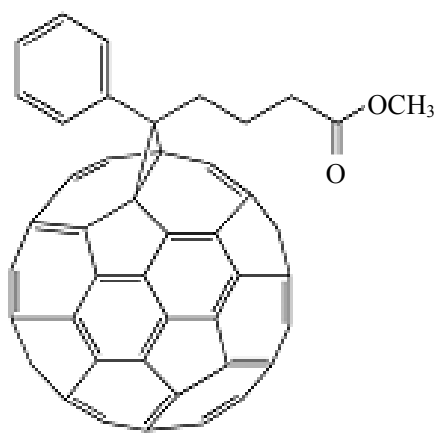

(a)

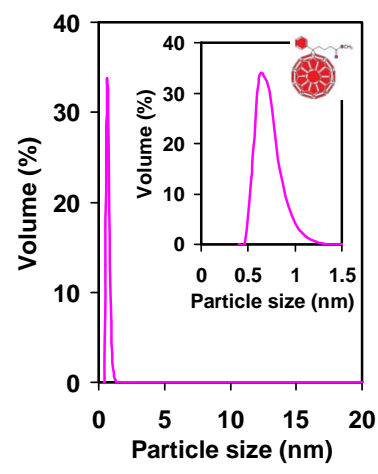

(b)

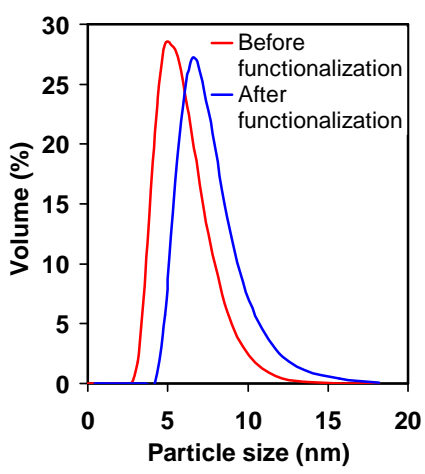

(b)

Figure 1. (a) Chemical structure of fullerene derivative PCBM used to form SAMs on AuNPs; (b) DLS showing the particle size distributions of PCBM $(0.8 \pm 0.1 \mathrm{~nm})$, as-synthesized AuNPs $(5.6 \pm 0.6 \mathrm{~nm})$, and PCBM-capped AuNPs $(7.3 \pm 0.7 \mathrm{~nm})$ in toluene, consistent with a single PCBM monolayer-protected AuNPs. 
Experiment section. The particle size distributions of PCBM and AuNPs (before and after PCBM functionalization) in toluene were determined using DLS, Figure 1(b). The sizes are $\sim 0.8 \mathrm{~nm}$ (PCBM), $\sim 5.6 \mathrm{~nm}$ (as-synthesized AuNPs), and $7.3 \mathrm{~nm}$ (PCBM-capped AuNPs), consistent with a single PCBM monolayer-protected AuNPs.

XPS was used to characterize the binding of $\mathrm{C}_{60}$ to AuNPs and the oxidation state of gold in the nanoparticles. All spectra were calibrated using the carbon $(\mathrm{C} 1 \mathrm{~s})$ peak (284.6 eV) [14]. The high resolution XPS Au 4f spectra of the AuNPs before and after the PCBM functionalization are shown in Figure 2. For the AuNPs before the PCBM functionalization, the $\mathrm{Au} 4 \mathrm{f} 7 / 2$ and $4 \mathrm{f} 5 / 2$ doublet gave rise to peaks positioned at $83.7 \mathrm{eV}$ and $87.4 \mathrm{eV}$, respectively, consistent with the typical values for $\mathrm{Au}(0)[15,16]$. Bulk $\mathrm{Au}(0)$ atoms are known to have $\mathrm{Au} 4 \mathrm{f} 7 / 2$ binding energy (BE) of $84.0 \mathrm{eV}$ [14] and the negative BE shift could arise from the clean gold surface atoms which caused a shift to a lower BE (of up to -0.4 $\mathrm{eV}$ ) in comparison with bulk atoms [17]. The observed spin-orbit splitting of $3.7 \mathrm{eV}$ agrees well with the literature value [14]. For the AuNPs after the PCBM functionalization, the $\mathrm{Au}$ 4f peaks exhibit a noticeable positive shift of about $0.6 \mathrm{eV}$ yielding binding energies of 84.3 $\mathrm{eV}$ and $88.0 \mathrm{eV}$ for $\mathrm{Au} \mathrm{4f7/2}$ and $\mathrm{Au} 4 \mathrm{f5} / 2$, respectively. The binding energy of the $\mathrm{Au} 4 \mathrm{f} 7 / 2$ for $\mathrm{Au}(\mathrm{I})$ has been reported to be $\sim 84.3$ to $84.9 \mathrm{eV}$ [15-17]. The observed positive BE shift for AuNPs bound to PCBM indicates that $\mathrm{Au}$ atoms were oxidized upon binding to $\mathrm{C}_{60}$, resulting from the charge transfer from $\mathrm{Au}$ to $\mathrm{C}_{60}$ at the $\mathrm{Au} / \mathrm{C}_{60}$ interface $[18,19]$.

The PCBM functionalized AuNPs were further investigated using transmission electron microscopy techniques incorporating HRTEM, STEM, and EELS. In

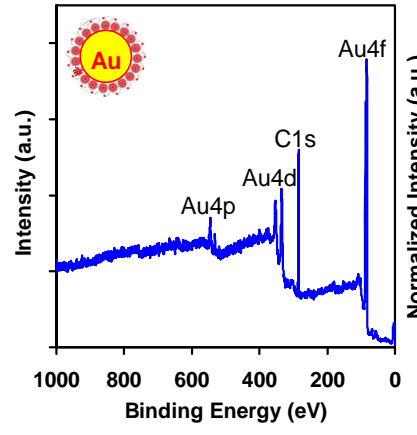

(a)

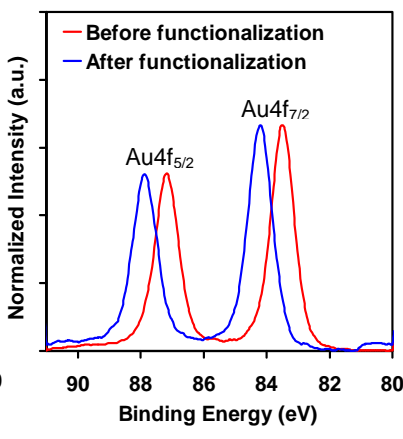

(b)
Figure 2. (a) XPS survey spectrum of the PCBM functionalized AuNPs; (b) High resolution XPS Au 4f spectra of AuNPs before and after the PCBM functionalization. Before functionalization, the binding energies of the $\mathrm{Au} 4 \mathrm{f} 7 / 2$ and $4 \mathrm{f5} / 2$ doublet are $83.7 \mathrm{eV}$ and $87.4 \mathrm{eV}$, respectively. After functionalization, a positive $\mathrm{BE}$ shift occurs resulting in binding energies of $84.3 \mathrm{eV}$ and $88.0 \mathrm{eV}$ for $\mathrm{Au} 4 \mathrm{f} 7 / 2$ and Au 4f5/2, respectively.
EELS, low energy loss spectra provide information about collective electronic as well as single particle/hole excitations associated with transitions between valence and conduction bands. Core loss EELS spectra obtained at higher energy losses can be analyzed to learn about the unoccupied density-of-states via energy-loss near-edge structure (ELNES) and local near neighbor coordination through extended energy-loss fine structure (EXELFS) [20]. HRTEM imaging reveals the presence of extensive periodicity associated with the SAMs of PCBM on AuNPs, Figure 3(a). The AuNPs were found to have a uniform size distribution of $\sim 5.5 \mathrm{~nm}$ (consistent with an average diameter of $5.6 \mathrm{~nm}$ obtained by DLS measurement in Figure 1(b)), and some of which exhibit the characteristic $\{111\}$ and $\{200\}$ lattice fringes. An interparticle spacing of $\sim 2 \mathrm{~nm}$ was observed, which indicates a single-monolayer of PCBM ( 1 nm in length) is on the AuNP surfaces (consistent with the result determined by DLS in Figure 1(b)). EELS in STEM line scan mode was employed to examine the PCBM functionalized AuNPs. Figure 3(b) shows the atomic-number $(Z)$ contrast STEM image of a discrete AuNP and the corresponding EELS low and core loss spectra at the edge of the AuNP surface. A beam diameter of $5 \AA$ was used to obtain the EELS data, which provides the high spatial resolution capability. The low loss EELS spectrum exhibits "peaks" at $\sim 6 \mathrm{eV}$ and $\sim 25 \mathrm{eV}$, in which the first peak was assigned to a collective excitation of $\pi$ elec-

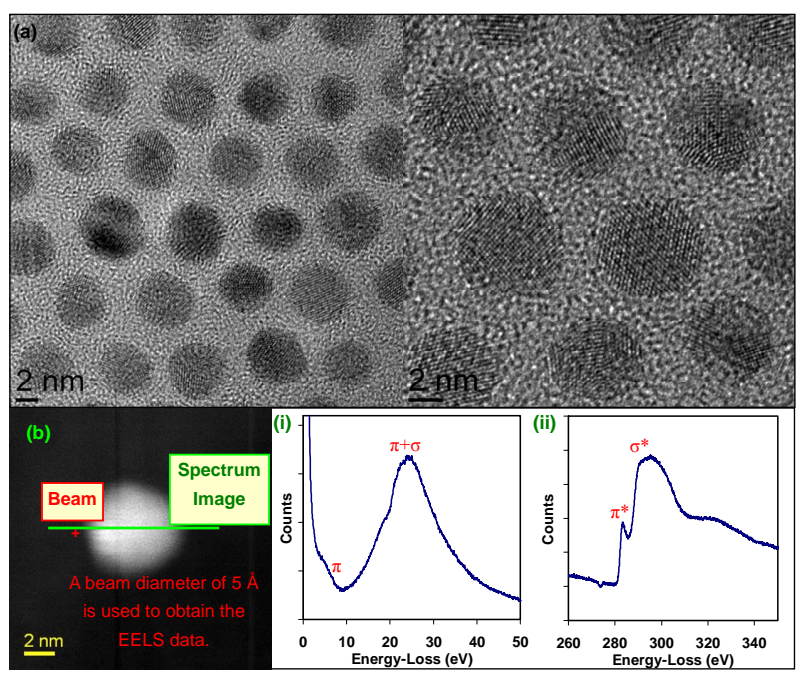

Figure 3. (a) Representative HRTEM micrographs showing the SAMs of PCBM on AuNPs. An interparticle spacing of $\sim 2 \mathrm{~nm}$ is observed, which indicates a single-monolayer of PCBM ( 1 nm in length) is on the AuNP surfaces; (b) Z-contrast STEM image of a discrete AuNP and the corresponding line scan EELS spectra at the edge of the AuNP surface, which signifies the presence of PCBM on the AuNP surface. EELS showing (i) the low energy loss spectrum with interband $\pi$ and $\pi+\sigma$ plasmons and (ii) the background subtracted $1 \mathrm{~s}$ core loss spectrum with a $\pi^{*}$ transition at $\sim 285 \mathrm{eV}$ and splitting of a $\sigma^{*}$ transition centered at $\sim 291 \mathrm{eV}$. 


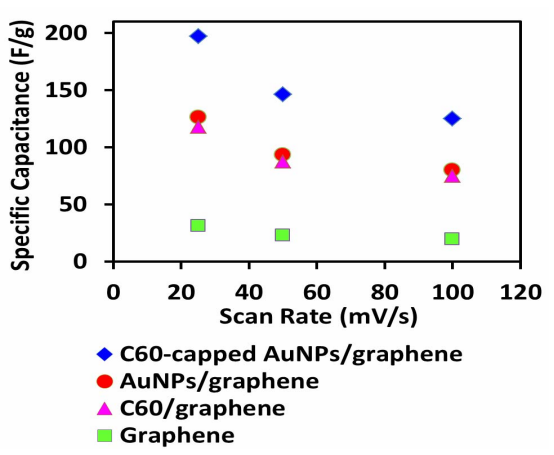

(a)

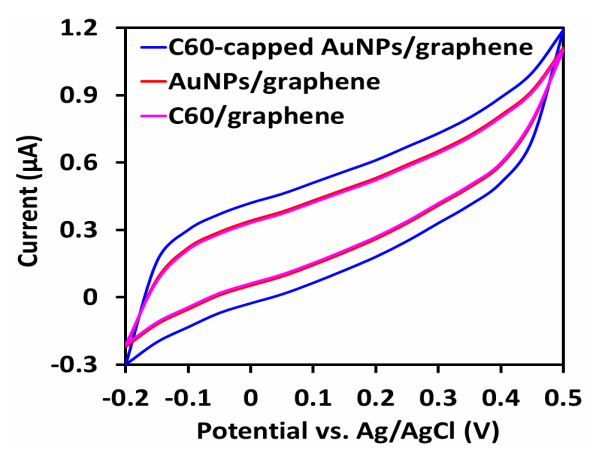

(b)

Figure 4. Comparison of electrochemical performance of graphene-based nanocomposites. (a) Specific capacitances of fullerene-capped AuNPs/graphene, AuNPs/graphene, fullerenes/graphene, and graphene electrodes in $0.5 \mathrm{M} \mathrm{KCl}$ buffer at scan rates of 25, 50, and $100 \mathrm{mV} / \mathrm{s}$; (b) Cyclic voltammograms of fullerene-capped AuNPs/graphene, AuNPs/ graphene, and fullerenes/graphene electrodes at a scan rate of $50 \mathrm{mV} / \mathrm{s}$.

trons (i.e. an interband $\pi$ plasmon) and the second broad peak was typically associated with a $\pi+\sigma$ plasmon, Figure 3(b-i). ELNES $1 \mathrm{~s}$ features can be utilized as fingerprints of $\mathrm{C}_{60}$ at high spatial resolution $(<2 \mathrm{~nm})$. Neglecting the core-hole effects, the two common features may be interpreted in terms of $\pi^{*}$ and $\sigma^{*}$ excitations for the first and second peaks, respectively. The background subtracted $1 \mathrm{~s}$ core loss spectrum exhibits a $\pi^{*}$ transition at $\sim 285 \mathrm{eV}$ and splitting of a $\sigma^{*}$ transition centered at $\sim 291 \mathrm{eV}$, consistent with the fingerprints of $\mathrm{C}_{60}$ [21] and hence confirming the presence of PCBM on the AuNP surface, Figure 3(b-ii).

Cyclic voltammetry was used to examine the electrochemical properties, Figure 4. Figure 4(a) shows the specific capacitance of PCBM-capped AuNPs/graphene nanocomposite film at various scan rates. The specific capacitances of AuNPs/graphene, PCBM/graphene, and graphene films were also determined for comparison. Their cyclic voltammograms are shown in Figure 4(b). The specific capacitance of PCBM-capped AuNPs/graphene electrode is $197 \mathrm{~F} / \mathrm{g}$, which is higher than that of graphene electrode (31 F/g), AuNPs/graphene electrode
(126 F/g), and PCBM/graphene electrode (118 F/g). The fullerene-capped AuNPs effectively separated the graphene sheets preventing aggregation - this accounts for the observed synergistic effect.

\section{Conclusion}

In summary, we have demonstrated that fullerene-capped AuNPs are effective spacing materials of graphene sheets for supercapacitor electrodes. Our fullerene-capped AuNPs/ graphene nanocomposite material exhibits excellent electrochemical properties with substantially enhanced capacitance performance when compared with the AuNPs/ graphene and fullerenes/graphene electrodes. The capacitance might be further improved by optimizing the size of gold nanoparticles and/or the mass ratio between graphene and fullerene-capped AuNPs. The results render a novel route of synthesis and modification of graphene-based materials for the construction of electrochemical energy storage devices.

\section{Acknowledgements}

The authors would like to thank the National Science Foundation and Department of Energy for financial support.

\section{REFERENCES}

[1] H. W. Kroto, J. R. Heath, S. C. Obrien, R. F. Curl and R. E. Smalley, "C $\mathrm{C}_{60}$ : Buckminsterfullerene," Nature, Vol. 318, No. 6042, 1985, pp. 162-163. doi: $10.1038 / 318162 \mathrm{a} 0$

[2] K. S. Novoselov, A. K. Geim, S. V. Morozov, D. Jiang, Y. Zhang, S. V. Dubonos, I. V. Grigorieva and A. A. Firsov, "Electric Field Effect in Atomically Thin Carbon Films," Science, Vol. 306, No. 5696, 2004, pp. 666-669. doi:10.1126/science.1102896

[3] V. Yong and J. M. Tour, "Theoretical Efficiency of Nanostructured Graphene-Based Photovoltaics," Small, Vol. 6, No. 2, 2010, pp. 313-318. doi:10.1002/smll.200901364

[4] D. Yu and L. Dai, "Self-Assembled Graphene/Carbon Nanotube Hybrid Filmsfor Supercapacitors," Journal of Physical Chemistry Letters, Vol. 1, No. 2, 2010, pp. 467470. doi:10.1021/jz9003137

[5] L. L. Zhang, R. Zhou and X. S. Zhao, "Graphene-Based Materials as Supercapacitor Electrodes," Journal of Materials Chemistry, Vol. 20, No. 29, 2010, pp. 5983-5992. doi: $10.1039 / \mathrm{c} 000417 \mathrm{k}$

[6] M. D. Stoller, S. J. Park, Y. W. Zhu, J. H. An and R. S. Ruoff, "Graphene-Based Ultracapacitors," Nano Letters, Vol. 8, No. 10, 2008, pp. 3498-3502. doi: $10.1021 / \mathrm{n} 1802558 \mathrm{y}$

[7] J. C. Love, L. A. Estroff, J. K. Kriebel, R. G. Nuzzo and G. M. Whitesides, "Self-Assembled Monolayers of Thiolates on Metals as a Form of Nanotechnology," Chemical Reviews, Vol. 105, No. 4, 2005, pp. 1103-1169. doi:10.1021/cr0300789 
[8] B. Bhushan, "Springer Handbook of Nanotechnology," 3rd Edition, Springer, Berlin, 2010. doi:10.1007/978-3-642-02525-9

[9] H. Park, J. Park, A. K. L. Lim, E. H. Anderson, A. P. Alivisatos and P. L. McEuen, "Nanomechanical Oscillations in a Single- $\mathrm{C}_{60}$ Transistor," Nature, Vol. 407, No. 6800, 2000, pp. 57-60. doi:10.1038/35024031

[10] G. J. Bubnis, S. M. Cleary and H. R. Mayne, "Self-Assembly and Structural Behavior of a Model Rigid $\mathrm{C}_{60^{-}}$ Terminated Thiolate on Au(111)," Chemical Physics Letters, Vol. 470, No. 4-6, 2009, pp. 289-294. doi:10.1016/j.cplett.2009.01.071

[11] L. Buglione, A. Bonanni, A. Ambrosi and M. Pumera, "Gold Nanospacers Greatly Enhance the Capacitance of Electrochemically Reduced Graphene," ChemPlusChem, Vol. 77, No. 1, 2012, pp. 71-73. doi:10.1002/cplu.201100016

[12] R. Klajn, M. A. Olson, P. J. Wesson, L. Fang, A. Coskun, A. Trabolsi, S. Soh, J. F. Stoddart and B. A. Grzybowski, "Dynamic Hook-and-Eye Nanoparticle Sponges," Nature Chemistry, Vol. 1, No. 9, 2009, pp. 733-738. doi:10.1038/nchem.432

[13] V. Yong and H. T. Hahn, "Graphene Growth with Giant Domains Using Chemical Vapor Deposition," CrystEngComm, Vol. 13, No. 23, 2011, pp. 6933-6936. doi:10.1039/c1ce05714f

[14] J. F. Moulder, W. F. Stickle, P. E. Sobol and K. D. Bomben, "Handbook of X-Ray Photoelectron Spectroscopy," Physical Electronics Division, Perkin-Elmer Corp., Norwalk, 1995.

[15] J. C. Zhou, X. H. Wang, M. Xue, Z. Xu, T. Hamasaki, Y.
Yang, K. Wang and B. Dunn, "Characterization of Gold Nanoparticle Binding to Microtubule Filaments," Materials Science \& Engineering C, Vol. 30, No. 1, 2010, pp. 20-26. doi:10.1016/i.msec.2009.08.003

[16] M. Brust, M. Walker, D. Bethell, D. J. Schiffrin and R. Whyman, "Synthesis of Thiol-Derivatized Gold Nanoparticles in a 2-Phase Liquid-Liquid System," Journal of the Chemical Society, Chemical Communications, No. 7, 1994, pp. 801-802. doi:10.1039/c39940000801

[17] M. C. Bourg, A. Badia and R. B. Lennox, "Gold-Sulfur Bonding in 2D and 3D Self-Assembled Monolayers: XPS Characterization," Journal of Physical Chemistry B, Vol. 104, No. 28, 2000, pp. 6562-6567. doi:10.1021/jp9935337

[18] M. Brust, C. J. Kiely, D. Bethell and D. J. Schiffrin, " $\mathrm{C}_{60}$ Mediated Aggregation of Gold Nanoparticles," Journal of the American Chemical Society, Vol. 120, No. 47, 1998, pp. 12367-12368. doi:10.1021/ja982776u

[19] J. T. Lyon and L. Andrews, "Infrared Spectrum of the $\mathrm{Au}-\mathrm{C}_{60}$ Complex," ChemPhysChem, Vol. 6, No. 2, 2005, pp. 229-232. doi:10.1002/cphc.200400467

[20] V. J. Keast, A. J. Scott, R. Brydson, D. B. Williams and J. Bruley, "Electron Energy-Loss Near-Edge Structure-A Tool for the Investigation of Electronic Structure on the Nanometre Scale," Journal of Microscopy, Vol. 203, No. 2, 2001, pp. 135-175. doi:10.1046/j.1365-2818.2001.00898.x

[21] V. P. Dravid, S. Z. Liu and M. M. Kappes, "Transmission Electron Microscopy of Chromatographically Purified Solid State $\mathrm{C}_{60}$ and $\mathrm{C}_{70}$," Chemical Physics Letters, Vol. 185, No. 1-2, 1991, pp. 75-81. doi:10.1016/0009-2614(91)80143-L 